Pacific Journal of Mathematics

AN EXISTENCE THEOREM FOR A GOURSAT PROBLEM 


\title{
AN EXISTENCE THEOREM FOR A GOURSAT PROBLEM
}

\author{
D. H. TUCKER
}

Introduction. Diaz [2] has established some global existence theorems concerning the partial differential equation $u_{x y}=f\left(x, y, u, u_{x}, u_{y}\right)$ by an analogue of the Euler-Cauchy polygon method, requiring that $u\left(x, y_{0}\right)=$ $\sigma(x)$ and $u\left(x_{0}, y\right)=\tau(y)$ where $\sigma$ and $\tau$ are of class $C^{\prime}$ on the ranges considered and $\sigma\left(x_{0}\right)=\tau\left(y_{0}\right), f(x, y, z, p, q)$ is a real valued function defined for all $(x, y, z, p, q)$ for which $x_{0} \leqq x \leqq x_{0}+a ; y_{0} \leqq y \leqq y_{0}+b,-\infty<z, p, q<\infty$, is continuous and bounded over that set, and satisfies a Lipschitz condition in the last two variables.

A local existence theorem is given here in which the boundary functions have a Lipschitz condition imposed rather than being of class $C^{\prime}$, and $f(x, y, z, p, q)=g(x, y, z) p+h(x, y, z) q+j(x, y, z)$ is required to be continuous over a more restricted set than that used by Diaz resulting in the convergence of the approximating functions over a subset of the original set rather than over the entire set defined by $x_{0} \leqq x \leqq x_{0}+a$; $y_{0} \leqq y \leqq y_{0}+b$. The fact that the boundary functions need not have first derivatives defined over their entire domains of definition results in the limit function not necessarily having first partial derivatives, nor a cross derivative defined over its entire domain of definition. The notion of an ordinary derivative [7] for an interval function is used to replace the cross derivative.

This is a correction of a stronger result announced earlier by the author (Abs. 550-15, Notices, AMS No. 1958).

Most of the material in $\S 1$ can be pieced together from the literature [3], [4], [5], [6]. The definitions and theorems needed will be stated here for convenience and in some cases proofs will be outlined as details in the method of proof are needed later in the paper and in some cases notation to be used later is established.

1. Preliminary definitions and theorems. By line interval will be meant a closed interval $a \leqq x \leqq b$. By plane interval will be meant a rectangular disk $a \leqq x \leqq b ; c \leqq y \leqq d$. These will be denoted by $[a, b]$ and $[a, b ; c, d]$. Suppose $g(x, y)$ is a function defined over $[A, B ; C, D]$. We define an interval function $G$ from $g$ as follows. Suppose $I=$ $[a, b ; c, d] \subset[A, B ; C, D]$, then $G(I)=\left.\left.g\right|_{a} ^{b}\right|_{c} ^{d}=g(a, c)-g(b, c)-g(a, d)+g(b, d)$ (with a similar notation $\left.G(I)\right|_{x=a}=g(a, c)-g(a, d)$ etc., for a function of a single variable). $G$ is an additive function. In what follows it is assumed that all points and intervals considered are in $[A, B ; C, D]$. It is easily seen that adding to $g$ functions of the single variable $x$ or $y$ does

Received May 16, 1961. 
not change $G$. It will be convenient to do this at times.

We shall say $g$ is nondecreasing if $G(I) \geqq 0$ for all $I$ and of bounded variation if $G$ is of bounded variation. It is not difficult to show that if $g$ is of bounded variation then it is the difference of two nondecreasing functions and conversely. We will say that $g$ is anchored if on some pair of lines $x=a, y=b, g=0$. If $g$ is nondecreasing and anchored then for each point $P(x, y)$ the eight limits $g(x+, y), g(x+, y+), g(x+, y-)$, $g(x-, y), g(x-, y+), g(x-, y-), g(x, y-)$, and $g(x, y+)$ exist and the set of all points for which any of these limits differ from $g(x, y)$ can be covered by a countable collection of line intervals each of which is parallel to one of the axes. If $g$ is of bounded variation and for $x=a$ and $y=b$, $g(\alpha, y)$ and $g(x, b)$ are of bounded variation then $g(\alpha, y)$ and $g(x, \beta)$ are of bounded variation for each $\alpha$ and each $\beta . G$ is said to satisfy a Lipschitz condition if there exists a number $M$ such that $|G(I) / \Delta I| \leqq M$ for all $I . \quad g$ is absolutely continuous means $G$ is absolutely continuous. A Lipschitz condition implies absolute continuity which implies bounded variation.

From McShane: A sequence of measurable sets $E_{1}, E_{2}, \cdots$ converges regularly to a point $x_{0}$ if there exists a set $\left\{Q_{n}\right\}$ of cubes (squares in our case) with centers at $x_{0}$ and possessing the following properties. For each $n$, the cube $Q_{n}$ contains the set $E_{n}$. As $n$ tends to $\infty$, the side of $Q_{n}$ tends to zero. There is a positive number $\alpha$ such that $m E_{n} \geqq \alpha m Q_{n}$ for each $n . \quad \alpha$ is called the modulus of regularity. We will be concerned of course with the case in which $E_{1}, E_{2}, \ldots$ are plane intervals. A Vitali covering theorem follows.

We now define the ordinary derivative of an interval function which we can apply to $G$ and associate with $g_{x y}$ in a certain way. Let $\phi(E)$ be a function of sets which is defined for all closed intervals contained in an interval $I_{0}$. Let $x_{0}$ be a point of $I_{0}$. The ordinary upper derivate $\bar{D} \phi\left(x_{0}\right)$ and the ordinary lower derivate $\underline{D} \phi\left(x_{0}\right)$ of $\phi$ at $x_{0}$ are respectively the greatest and the least of all numbers $l$ for which there exists a sequence $\left\{I_{n}\right\}$ of closed subintervals of $I_{0}$ containing $x_{0}$, converging regularly to $x_{0}$ and having limit $\left[\phi\left(I_{n}\right) / \Delta I_{n}\right]=l$. If $\underline{D} \phi\left(x_{0}\right)=\bar{D} \phi\left(x_{0}\right)$ then this number is denoted $D \phi\left(x_{0}\right)$ and is called the ordinary derivative of $\phi$ at $x_{0}$. By the ordinary derivative of $g$ at the point $\left(x_{0}, y_{0}\right)$ will be meant $D G\left(x_{0}, y_{0}\right)$ and will be denoted $D g\left(x_{0}, y_{0}\right)$. If $g$ possesses a cross derivative $g_{x y}$ in some open neighborhood of $\left(x_{0}, y_{0}\right)$ which is continuous at $\left(x_{0}, y_{0}\right)$ then $D g\left(x_{0}, y_{0}\right)=g_{x y}\left(x_{0}, y_{0}\right)$.

THEOREM 1.1. If $g$ is of bounded variation over the plane interval $I$ the $D g$ exists almost everywhere on $I$.

THeOREM 1.2. If $g$ satisfies a Lipschitz condition with constant $M$ over $I=[a, b ; c, d]$ then $D g$ is Lebesque integrable over $I$ and if $g$ is 
also continuous over $I$, then $g(x, y)=g(x, c)+g(a, y)-g(a, c)+\int_{a}^{x} \int_{c}^{y} D g$.

An outline of a proof will be given since the method of proof here motivates the procedure of $\S 2$.

It is sufficient to show that there exists a sequence $\left\{h^{n}\right\}$ of step functions which converge to $D g$ a.e. on $I$ and are such that $\left\{\int_{a}^{x} \int_{c}^{y} h^{n}\right\}$ forms an equi-absolutely continuous collection over $I,[7$, p. 171] since $D g \leqq M$ on $I$. For each positive integer $n$ subdivide each of $[a, b]$ and $[c, d]$ into $n$ subintervals of equal length. We now have $n^{2}$ congruent subintervals $\left\{I_{i j}^{n}\right\}$ for each $n$ which are similar for all $n$. Define

$$
\begin{aligned}
h^{n}(x, y) & =G\left(I_{i j}^{n}\right) / \Delta I_{i j}^{n} \text { if }(x, y) \text { is interior to } I_{i j}^{n} \\
& =0 \text { elsewhere. }
\end{aligned}
$$

It follows at once that $\int_{a}^{x} \int_{c}^{y} h^{n}$ satisfy a uniform Lipschitz constant (namely $M$ ) and hence are equi-absolutely continuous and that $h^{n} \rightarrow D g$ a.e. on $I$. In order to get the formula of the theorem we adjoin boundary terms to $h^{n}$ in the following way.

$$
\begin{aligned}
f^{n}(x, y)= & g\left(x_{i-1}, y_{j-1}\right)+\left[\left.G\left(I_{i j}^{n}\right)\right|_{x=x_{i-1}} \mid \Delta y\right]\left(y-y_{j-1}\right) \\
& +\left[\left.G\left(I_{i j}^{n}\right)\right|_{y=y_{j-1}} \mid \Delta x\right]\left(x-x_{i-1}\right)+h^{n}(x, y)\left(x-x_{i-1}\right)\left(y-y_{j-1}\right) \\
f^{n}(x, y)= & P_{1} g(x, c)+P_{2} g(a, y)-g(a, c)+\int_{a}^{x} \int_{c}^{y} h^{n}
\end{aligned}
$$

where $P_{1} g(x, c)=\left(\left[g\left(x_{i}, c\right)-g\left(x_{i-1}, c\right)\right] / \Delta x\right)\left(x-x_{i-1}\right)+g\left(x_{i-1}, c\right)$

$$
\begin{aligned}
& x_{i-1} \leqq x \leqq x_{i} \\
P_{2} g(a, y)= & \left(\left[g\left(a, y_{j}\right)-g\left(a, y_{j-1}\right)\right] / \Delta y\right)\left(y-y_{j-1}\right)+g\left(a, y_{j-1}\right) \\
& y_{j-1} \leqq y \leqq y_{j} .
\end{aligned}
$$

$f^{n}$ converges uniformly to $g$ and $P_{1} g(x, c)$ and $P_{2} g(a, y)$ converge uniformly to $g(x, c)$ and $g(a, y)$.

In order to treat the problem at hand some consideration must be given to first partial derivatives. The following theorem will prove useful.

Theorem 1.3. Suppose $I=[a, b ; c, d]$ is an interval, $g$ satisfies $a$ Lipschitz condition over $I, g(a, y)$ and $g(x, c)$ are of bounded variation in $y$ and $x$ respectively. $g_{x}$ and $g_{y}$ exist a.e. on $I$.

Inasmuch as the author knows of no reference to this theorem an outline of a proof is given. Taking into account the remarks in the first paragraph of this section, one need consider only an anchored nondecreasing function over $I$ which satisfies a Lipschitz condition. There is no loss in supposing $I$ in the first quadrant. For each rational $r$ in $[c, d] g_{x}(x, r)$ exists a.e. on $[a, b]$. Denote the union of the exceptional sets on $[a, b]$ for these rationals by $S$. $S$ has length zero. It follows now that for 
each $t$ in $[a, b] g_{x}(t, y)$ is nondecreasing over the set of $[c, d]$ for which it is defined. Suppose now $t$ is in $[a, b]-S$, then $g_{x}(t, y)$ exists for all rational $y$. Suppose $\bar{y}$ is not rational, then from the quasi-continuity of $g_{x}(t, y)$ for each $t$ and the Lipschitz condition on $g$ it follows that $g_{x}(t, \bar{y})$ exists. Hence, there are no further exceptional points and the set of all exceptional points is of plane measure zero.

2. An existence theorem. Suppose $a$ and $b$ are functions defined over $0 \leqq x \leqq X, 0 \leqq y \leqq Y$ respectively, not both the zero function, $a(0)=b(0)=c$ and each of $a$ and $b$ satisfy a Lipschitz condition with constant $K>0$. Denote by $k$ the max absolute value of $a$ and $b$ on their ranges. Suppose there exists a number $\infty>\lambda>1$ such that $g(x, y, z), h(x, y, z)$ and $j(x, y, z)$ are continuous over the interval $[0, X ; 0, Y ;-\lambda k, \lambda k]$. For convenience write

$$
f(x, y, z, p, q)=g(x, y, z) p+h(x, y, z) q+j(x, y, z) .
$$

Denote by $R$ the interval

$$
[0, X ; 0, Y ;-\lambda k, \lambda k ;-(K+L),(K+L) ;-(K+L),(K+L)]
$$

for some $0<L<\infty$, and by $M$ the $\max |f|$ over $R$. Consider the planes $P_{1}:(K+L) x+k=z, \quad P_{2}:(K+L) x+k=-z, \quad P_{3}:(K+L) y+k=$ $z, \quad P_{4}:(K+L) y+k=-z$ and their intersections $x=(\lambda-1) k /(K+L)$; $y=(\lambda-1) k /(K+L)$ with the planes $z=\lambda k$ and $z=-\lambda k$ over the first quadrant. Now choose $A$ so that $A \leqq \min \{X,(\lambda-1) k /(K+L)\}$ and $A M \leqq L$; and $B$ so that $B \leqq \min \{Y,(\lambda-1) k /(K+L)\}$ and $B M \leqq L$.

THEOREM 2.1. There exists a function $u$ defined on $[0, A ; 0, B]$ which is continuous and satisfies a Lipschitz condition on $[0, A ; 0, B]$ and such that $u(0, y)=b(y) ; u(x, 0)=a(x)$ and

$$
D u(x, y)=g(x, y, u) u_{x}+h(x, y, u) u_{y}+j(x, y, u) \text { a.e. on }[0, A ; 0, B] \text {. }
$$

Proof. The proof is by construction. A sequence of functions will be constructed, much like the procedure in Theorem 1.2, the sequence being uniformly bounded, equi-continuous and satisfying a uniform Lipschitz condition. Ascoli's theorem asserts the existence of a uniformly convergent subsequence and then it will be shown that the limit of such a subsequence is indeed a solution.

For each positive integer $n$ consider the sequences $\{j A / n\}_{j=0}^{n}=\{\tilde{j}\}_{j=0}^{n}$ and $\{k B / n\}_{k=0}^{n}=\left\{\frac{1}{k}\right\}_{k=0}^{n}$ which subdivide $[0, A ; 0, B]$ into $n^{2}$ congruent subintervals $\left\{I_{j, k}^{n}\right\}$ for each $n$ which are similar for all $n$. Now for each $n$ a function $u^{n}$ is defined by recurrence relations in the following way. First $u^{n}$ is defined along the axes in such a way that $u^{n}(x, 0)$ is a polygonal graph of chords joining consecutive points $(\tilde{j}, a(\tilde{j}))$ of the graph of $a$ and a similar definition holds for $u^{n}(0, y)$ with respect to $b . u^{n}$ is next defined over $I_{11}^{n}$ as a ruled surface 


$$
u^{n}(x, y)=c+m_{1}(x-\tilde{0})+m_{2}(y-\stackrel{\perp}{0})+f\left(\tilde{0}, \stackrel{\frac{1}{0}}{0}, c, m_{1}, m_{2}\right)(x-\tilde{0})(y-\stackrel{\perp}{0})
$$

where $m_{1}$ is the slope of the chord joining $(0, c)$ and $(\tilde{1}, a(\tilde{1}))$ and similarly for $m_{2}$. The procedure continues as follows. Suppose for a given interval $I_{j k}^{n} u^{n}$ has been defined along the "aft" sides, $x=\widetilde{j-1}, y=\frac{1}{k-1}$, then over $I_{k j}^{n}$

$$
\begin{aligned}
& u^{n}(x, y)=u^{n}\left(\widetilde{j-1}, \frac{1}{k-1}\right)+m_{1}(x-\widetilde{j-1})+m_{2}\left(y-\frac{1}{k-1}\right) \\
+ & f\left(\widetilde{j-1}, \frac{1}{k-1}, u^{n}\left(\widetilde{j-1}, \frac{1}{k-1}\right), m_{1}, m_{2}\right)(x-j-1)\left(y-\frac{1}{k-1}\right)
\end{aligned}
$$

where $m_{1}$ and $m_{2}$ are the slopes (already determined from previous computations) along the "aft" $x$ and $y$ sides of $I_{j k}^{n}$ respectively.

For each $n$, the graph of $u^{n}$ consists of $n^{2}$ ruled surfaces, each of which is ruled in both the $x$ and $y$ directions, the adjacent ruled surfaces having common line intervals on their boundaries. These surfaces cannot have extremum except on their boundary sets, hence if it can be shown that the boundary sets are uniformly bounded it will follow that $\left\{u^{n}\right\}$ is uniformly bounded.

Denote by $D$ the domain bounded by the planes $P_{1}, P_{2}, P_{3}, P_{4}, y=0$, $x=0, x=A$ and $y=B$. Suppose $n$ is a positive integer and consider the boundary pieces of $u^{n}$ on $I_{11}^{n}$, for $x=\tilde{0}, y=\stackrel{\perp}{0}, x=\tilde{1}$ and $y=\frac{1}{1}$ respectively we have for the slopes

$$
\begin{aligned}
& \left|m_{2}\right|=\left|\left[b\left(\frac{1}{1}\right)-b\left(\frac{1}{0}\right)\right] / \frac{1}{1}\right|<K<K+L \\
& \left|m_{1}\right|=|[a(\tilde{1})-a(\tilde{0})] / \tilde{1}|<K<K+L \\
& \left|m_{2}+f\left(\tilde{0}, \stackrel{+}{0}, c, m_{1}, m_{2}\right) x\right|<K+M \cdot \tilde{1}<K+M A<K+L \\
& \left|m_{1}+f\left(\tilde{0}, \stackrel{\perp}{0}, c, m_{1}, m_{2}\right) y\right|<K+M \cdot \stackrel{\perp}{1}<K+M B<K+L
\end{aligned}
$$

hence, since the pieces start in $D$, they must remain in $D$. It is easily seen that the boundary pieces along the axes are in $D$ since all have slopes bounded by $K$, therefore it is sufficient new to investigate only the "fore" boundaries of each interval. Consider the interval $I_{j k}^{n}$ and denote by $m_{1}(j, k)$ and $m_{2}(j, k)$ the slopes of the boundaries along $y=\frac{1}{k}$ and $x=\tilde{j}$. Suppose that the relationship $\left|m_{1}(p, q)\right|<K+M \stackrel{\perp}{q}$ holds for $p=0,1, \ldots, j ; q=0,1, \ldots, k-1$ and $\left|m_{2}(p, q)\right|<K+\tilde{M p}$ holds for $p=0,1, \ldots, j-1 ; q=0,1, \ldots, k$. We have just shown that these do for $p=q=1$ and along the axes it was observed that $K$ would suffice for a bound.

$$
\begin{aligned}
& \quad m_{2}(j, k)=m_{1}(j, k-1) \\
& +f\left(\widetilde{j-1}, \frac{1}{k-1}, u^{n}\left(\widetilde{j-1}, \frac{1}{k-1}\right), m_{1}(j, k-1), m_{2}(j-1, k)\right)\left(y-\frac{1}{k-1}\right)
\end{aligned}
$$

and 


$$
\begin{gathered}
m_{2}(j, k)=m_{2}(j-1, k) \\
+f\left(\widetilde{j-1}, \frac{1}{k-1}, u^{n}\left(\widetilde{j-1}, \frac{1}{k-1}\right), m_{1}(j, k-1) m_{2}(j-1, k)\right)(x-\widetilde{j-1})
\end{gathered}
$$

hence

$\left|m_{2}(j, k)\right| \leqq\left|m_{1}(j, k-1)\right|+M \cdot \stackrel{\perp}{1} \leqq K+M\left(\frac{1}{k-1}\right)+M \cdot \stackrel{\perp}{1}=K+\frac{1}{M k}<K+L$ and

$\left|m_{1}(j, k)\right| \leqq\left|m_{2}(j-1, k)\right|+M \cdot \tilde{1} \leqq K+M(\widetilde{j-1})+M \cdot \tilde{1}=K+M \tilde{j}<K+L$,

thus the induction is complete, the slopes of the boundary pieces are bounded by $K+L$ and hence since $u^{n}$ starts in $D$ it must remain there for each $n$ and therefore $\left\{u^{n}\right\}$ is uniformly bounded.

It follows easily from this argument that $u^{n}$ cannot have a slope in either the $x$ or $y$ directions as great as $K+L$, and this gives the equicontinuous property at once. Suppose $n$ is an integer, $e>0$ and $d=$ $e / 2(K+L),(a, b)$ and $(p, q)$ are in $[0, A ; 0, B] ;|a-p|<d$ and $|b-q|<d$. Then

$$
\begin{gathered}
\left|u^{n}(a, b)-u^{n}(p, q)\right| \leqq\left|\left[u^{n}(a, b)-u^{n}(a, q)\right](b-q) /(b-q)\right| \\
\quad+\mid\left[u^{n}(a, q)-u^{n}(p, q)(a-p)(a-p) \mid<2(K+L) d=e,\right.
\end{gathered}
$$

which establishes the property.

Let $V\left[I_{j k}^{n}, u^{n}\right]$ denote the total variation of $u^{n}$ over $I_{j k}^{n}$. It is easily seen that

$$
V\left[I_{j k}^{n}, u^{n}\right]=\mid f\left(\overparen{j-1}, \frac{1}{k-1}, u^{n}\left(\widetilde{j-1}, \frac{1}{k-1}\right),\right.
$$

$\left.m_{1}(j, k-1), m_{2}(j-1, k)\right) \mid \cdot \tilde{1} \cdot \frac{1}{1} \leqq M \cdot \tilde{1} \cdot \frac{1}{1}$ so that $V\left[[0, A ; 0, B], u^{n}\right] \leqq M A B$ for all $n$, hence $\left\{u^{n}\right\}$ is uniformly of bounded variation. A closer look at the preceding statement shows that for any subinterval $I=[a, b ; c, d]$ of $[0, A ; 0, B] V\left[[a, b ; c, d], u^{n}\right] \leqq M(b-a)(c-d)$ and hence $\left|U^{n}(I) / \Delta I\right| \leqq M$ and $\left\{u^{n}\right\}$ satisfies a uniform Lipschitz condition with constant $M$.

Now making use of Ascoli's theorem [1] there is an infinite subsequence $\left\{u^{n i}\right\}$ of $\left\{u^{n}\right\}$ which converges uniformly to a limit function on $[0, A ; 0, B]$. Denote one such subsequence by $\left\{u^{m}\right\}$ and its limit by $u$. Since each $u^{n}$ is continuous so is $u$. Furthermore $u$ satisfies a Lipschitz condition with constant $M, u(x, 0)=a(x)$ and $u(0, y)=b(y)$. From Theorem 1.2 we have

$$
u(x, y)=a(x)+b(y)-c+\int_{0}^{x} \int_{0}^{y} D u .
$$

We have yet to show that $D u=g(x, y, u) u_{x}+h(x, y, u) u_{y}+j(x, y, u)$ a.e. From Theorem 1.3 we know that $u_{x}$ and $u_{y}$ exist a.e. on $[0, A ; 0, B]$. Now recall that in the sequence $\left\{u^{n}\right\}$ and therefore in $\left\{u^{m}\right\}$ it was observed that no slope in the $x$ or $y$ directions could exceed $K+L$, therefore 
$\left|u_{x}\right| \leqq K+L,\left|u_{y}\right| \leqq K+L,\left|u_{x}^{m}\right| \leqq K+L$ and $\left|u_{y}^{m}\right| \leqq K+L$ where $u_{x}^{m}$ and $u_{y}^{m}$ are the slopes in the usual sense at points where the derivatives exist and are the average of the left and right limits elsewhere.

We first show that $u_{x}$ and $u_{y}$ are Lebesque integrable with respect to $x$ and $y$ respectively. It will suffice to show one, say $u_{x}$. Recall the technique of Theorem 1.3. Here the function was first anchored and then decomposed into nondecreasing functions. $u$ can be anchored on the axes by considering $\lambda(x, y)=u(x, y)-a(x)-b(y)+c$. The only effect of this on $u_{x}$ is the removal of $a^{\prime}(x)$ which is Lebesque integrable since $a$ is absolutely continuous. Recall that $u$ satisfies a Lipschitz condition, hence $\lambda$ satisfies the same condition (i.e., same Lipschitz constant) and is anchored, hence $\lambda$ is absolutely continuous in $x$ for each $y$ and $y$ for each $x$, therefore $u$ is also. Therefore $u(x, y)-u(0, y)=\int_{0}^{x} u_{x}(t, y) d t$ for each $y$ and similarly for $u_{y}$. Since $g$ and $h$ are continuous, $g u_{x}$ and $h u_{y}$ are integrable with respect to $x$ and $y$ respectively. It is easily seen that $u_{x}$ is the limit a.e. of a sequence of measurable functions on $[0, A ; 0, B]$ and hence is measurable and therefore since it is bounded it is integrable and $g u_{x}$ is also integrable and by Fubini's theorem

$$
\int_{0}^{y} \int_{0}^{x} g u_{x}=\int_{0}^{y}\left[\int_{0}^{x} g(t, z, u) u_{x}(t, z) d t\right] d z .
$$

Similarly for $u_{y}$ and hence $h u_{y}$

$$
\int_{0}^{x} \int_{0}^{y} h u_{y}=\int_{0}^{x}\left[\int_{0}^{y} h(t, z, u) u_{y}(t, z) d z\right] d t .
$$

Define, for fixed $(x, y)$, a functional $T(v)$ such that

$$
\begin{gathered}
T(v)=\int_{0}^{y}\left[\int_{0}^{x} g(t, z, u) v_{x}(t, z) d t\right] d z+\int_{0}^{x}\left[\int_{0}^{y} h(t, z, u) v_{y}(t, z) d z\right] d t \\
+\int_{0}^{y} \int_{0}^{x} j(t, z, u) d t d z
\end{gathered}
$$

and, again as in Theorem 1.2 write the functions $\left\{u^{m}\right\}$ in integral form,

$$
\begin{aligned}
u^{m}(x, y)= & P_{1}^{m} a(x)+P_{2}^{m} b(y)-c+\int_{0}^{x} \int_{0}^{y} f\left(\widetilde{j-1}, \frac{1}{k-1}, u^{m}\left(\widetilde{j-1}, \frac{1}{k-1}\right),\right. \\
& \left.m_{1}(j, k-1), m_{2}(j-1, k)\right) d t d z
\end{aligned}
$$

where $P_{1}^{m} a(x)$ and $P_{2}^{m} b(y)$ are the $m$ th polygonal approximations to $a$ and $b$ respectively. Recalling that $f=g u_{x}+h u_{y}+j$ we have,

$$
\begin{gathered}
u^{m}(x, y)=P_{1}^{m} a(x)+P_{2}^{m} b(y)-c+\int_{0}^{x} \int_{0}^{y} g\left(\widetilde{j-1}, \frac{1}{k-1}, u^{m}\left(\overparen{j-1}, \frac{1}{k-1}\right)\right) \\
m_{1}(j, k-1) d t d z+\int_{0}^{x} \int_{0}^{y} h\left(\widetilde{j-1}, \frac{1}{k-1}, u^{m}\left(\widetilde{j-1}, \frac{1}{k-1}\right)\right) m_{2}(j-1, k) d t d z \\
+\int_{0}^{x} \int_{0}^{y} j\left(\widetilde{j-1}, \frac{1}{k-1}, u^{m}\left(\widetilde{j-1}, \frac{1}{k-1}\right)\right) d t d z .
\end{gathered}
$$


Now define

$$
\begin{aligned}
v^{m}(x, y)=a(x) & +b(y)-c+\int_{0}^{x} \int_{0}^{y} g(t, z, u(t, z)) u_{x}^{m}(t, z) \\
& +h(t, z, u(t, z)) u_{y}^{m}(t, z)+j(t, z, u(t, z)) d z d t .
\end{aligned}
$$

Now consider

$$
\begin{aligned}
& \left|u^{m}-v^{m}\right| \leqq\left|P_{1}^{m} a-a\right|+\left|P_{2}^{m} b-b\right|+\int_{0}^{x} \int_{0}^{y}|g| \cdot \mid m_{1}(j, k-1) \\
& \quad-u_{x}^{m}(t, z) \mid d t d z+\int_{0}^{x} \int_{0}^{y} g\left(\widetilde{j-1}, \frac{1}{k-1}, u^{m}\left(\widetilde{j-1}, \frac{1}{k-1}\right)\right) \\
& \quad-g\left(t, z, u(t, z)|\cdot| u_{x}^{m}(t, z)\left|d t d z+\int_{0}^{x} \int_{0}^{y}\right| h|\cdot| m_{2}(j-1, k)-u_{y}^{m}(t, z) \mid d t d z\right. \\
& \quad+\int_{0}^{x} \int_{0}^{y}\left|h\left(\widetilde{j-1}, \frac{1}{k-1}, u^{m}\left(\widetilde{j-1}, \frac{1}{k-1}\right)\right)-h(t, z, u(t, z))\right| \cdot\left|u_{y}^{m}(t, z)\right| d t d z \\
& \quad+\int_{0}^{x} \int_{0}^{y}\left|j\left(\widetilde{j-1}, \frac{1}{k-1}, u^{m}\left(\widetilde{j-1}, \frac{1}{k-1}\right)\right)-j(t, z, u(t, z))\right| d t d z .
\end{aligned}
$$

Taking into account that $P_{1}^{m} a \rightarrow a, P_{2}^{m} b \rightarrow b, u^{m} \rightarrow u$ uniformly and that $g, h, j$ and $u$ are uniformly continuous and $u_{x}^{m}$ and $u_{y}^{m}$ are uniformly bounded we can restrict our attention to the terms involving

$$
\left|m_{1}(j, k-1)-u_{x}^{m}(t, z)\right| \text { and }\left|m_{2}(j-1, k)-u_{y}^{m}(t, z)\right| \text {. }
$$

Recalling the definition of $u^{m}(t, z)$ we have at once that each of these is uniformly less than $M \cdot \tilde{1}$ and $M \cdot \stackrel{\perp}{1}$ respectively and hence converge uniformly to zero. Hence we have that $\left|u^{m}-v^{m}\right| \rightarrow 0$ and hence $v^{m}(x, y) \rightarrow u(x, y)$, and hence that $T\left(u^{m}\right) \rightarrow \int_{0}^{x} \int_{0}^{y} D u$, for each $(x, y)$ in $[0, A ; 0, B]$.

We shall now show that $T\left(u^{m}\right) \rightarrow T(u)$ and hence obtain $g(x, y, u) u_{x}+$ $h(x, y, u) u_{y}+j(x, y, u)=D u$ a.e. Suppose $e>0$. Since $g$ and $h$ are continuous over $[0, A ; 0, B]$ they can be approximated by funcions $g_{p}$ and $h_{p}$ which are $C^{\prime}$ (and hence absolutely continuous in $x$ and in $y$ ) and are zero on the boundary of $[0, A ; 0, B]$ and such that

$$
\int_{0}^{x} \int_{0}^{y}\left|g-g_{p}\right|^{2}<e / 12(K+L)^{2} A B \text { and } \int_{0}^{x} \int_{0}^{y}\left|h-h_{p}\right|^{2}<e / 12(K+L)^{2} A B .
$$

Denote by $E$ a common bound on $\left|g_{p}\right|$ and $\left|h_{p}\right|$. Now consider

$$
\left|T\left(u^{m}\right)-T(u)\right| \leqq\left|T\left(u^{m}-u\right)-T_{p}\left(u^{m}-u\right)\right|+\left|T_{p}\left(u^{m}-u\right)-\iint j\right| .
$$

where $T_{p}$ is $T$ with $g$ and $h$ replaced by $g_{p}$ and $h_{p}$ respectively.

$$
\begin{gathered}
\left|T\left(u^{m}\right)-T(u)\right| \leqq\left|\int_{0}^{y}\left[\int_{0}^{x}\left(g-g_{p}\right)\left(u_{x}^{m}-u_{x}\right) d t\right] d z\right| \\
+\left|\int_{0}^{x}\left[\int_{0}^{y}\left(h-h_{p}\right)\left(u_{y}^{m}-u_{y}\right) d z\right] d t\right|+\left|\int_{0}^{y}\left[\int_{0}^{x} g_{p}\left(u_{x}^{m}-u_{x}\right) d t\right] d z\right| \\
+\left|\int_{0}^{x}\left[\int_{0}^{y} h_{p}\left(u_{y}^{m}-u_{y}\right) d z\right] d t\right|
\end{gathered}
$$


the terms in $j(x, y, u)$ adding to zero. In the first two terms on the right, apply the Schwartz inequality, recall that $\left|u_{x}^{m}-u_{x}\right|<2(K+L)$ and $\left|u_{y}^{m}-u_{y}\right|<2(K+L)$ and obtain $e / 3$ in each case for an upper bound. In each of the second two terms, (since $g_{p}$ and $h_{p}$ are absolutely continuous in $x$ and $y$ respectively) an integration by parts is carried out and we have

$$
\leqq 2 e / 3+\left|\int_{0}^{y}\left[\int_{0}^{x} g_{p_{x}}\left(u^{m}-u\right) d t\right] d z\right|+\left|\int_{0}^{x}\left[\int_{0}^{y} h_{p_{y}}\left(u^{m}-u\right) d z\right] d t\right|
$$

now since $u^{m} \rightarrow u$ uniformly, choose $N$ large enough that $\int_{0}^{y} \int_{0}^{x}\left|u^{m}-u\right|<e / 6 E$ for $m>N$ and obtain $\left|T\left(u^{m}\right)-T(u)\right|<e$ for $m>N$. This establishes the theorem.

REMARKs. The argument here depends heavily on the fact that, except for the term in $j(x, y, u), T$ is a linear functional. For example if $f(x, y, z, p, q)=g(x, y, z) p^{2}$, the argument fails. It also depends upon the fact that $u_{x}^{n}, u_{x}, u_{y}^{n}$ and $u_{y}$ all have a common bound and that the convergence of $u^{m}$ to $u$ is uniform. This indicates that there is little, if any, hope of using this technique to obtain global solutions for the quasi-linear equation. It is of interest to note, however, that the method can be extended to equations of higher order if the linearity conditions in $f$ are maintained and no derivatives of order greater than one occur in $f$.

\section{REFERENCES}

1. G. Ascoli, Le curve limite di una varietà data di curve, Memorie della Reale Accademı dei Lincei, Serie 3, 18, (1883), 521-586.

2. J. B. Diaz, On an Analogue of the Euler-Cauchy Polygon Method for the Numerical Solution of $u_{x y}=F\left(x, y, u, u_{x}, u_{y}\right)$, NAVORD Report 4451 (16 January 1957), White Oak, Maryland, U. S. Naval Ordnance Laboratory, also Arch. Rational Mechanics and Analysis, 1 (1958), 357-390.

3. M. Fréchet, Nouvelles Annales de Mathematicques, 10 (1910), 247-248

4. G. H. Hardy, On Double Fourier Series, J. Math., 37 (1906), 57-59.

5. E. W. Hobson, Functions of a Real Variable, 1, 3rd edition, Washington, D. C., Harren Press, 1927.

6. John Martin Krause, Mittlewertsatze im Gebiete d. Doppelsummen und Doppelintegral, Leipzig, Ges. d. Wiss. S. Ber., 55 (1903).

7. Edward J. McShane, J. Integration, New Jersey, Princeton University Press, 1944.

UNIVERSITY OF UTAH 



\title{
PACIFIC JOURNAL OF MATHEMATICS
}

\author{
EDITORS
}

Ralph S. Phillips

Stanford University

Stanford, California

M. G. Arsove

University of Washington

Seattle 5, Washington
A. L. Whiteman

University of Southern California Los Angeles 7, California

Lowell J. Paige

University of California

Los Angeles 24, California

\section{ASSOCIATE EDITORS}
E. F. BECKENBACH
D. DERRY
M. OHTSUKA
H. L. ROYDEN
E. SPANIER
E. G. STRAUS
T. M. CHERRY
F. WOLF

\section{SUPPORTING INSTITUTIONS}

\author{
UNIVERSITY OF BRITISH COLUMBIA \\ CALIFORNIA INSTITUTE OF TECHNOLOGY \\ UNIVERSITY OF CALIFORNIA \\ MONTANA STATE UNIVERSITY \\ UNIVERSITY OF NEVADA \\ NEW MEXICO STATE UNIVERSITY \\ OREGON STATE UNIVERSITY \\ UNIVERSITY OF OREGON \\ OSAKA UNIVERSITY \\ UNIVERSITY OF SOUTHERN CALIFORNIA
}

\author{
STANFORD UNIVERSITY \\ UNIVERSITY OF TOKYO \\ UNIVERSITY OF UTAH \\ WASHINGTON STATE UNIVERSITY \\ UNIVERSITY OF WASHINGTON \\ $*$
AMERICAN MATHEMATICAL SOCIETY \\ CALIFORNIA RESEARCH CORPORATION \\ SPACE TECHNOLOGY LABORATORIES \\ NAVAL ORDNANCE TEST STATION
}

Mathematical papers intended for publication in the Pacific Journal of Mathematics should be typewritten (double spaced), and the author should keep a complete copy. Manuscripts may be sent to any one of the four editors. All other communications to the editors should be addressed to the managing editor, L. J. Paige at the University of California, Los Angeles 24, California.

50 reprints per author of each article are furnished free of charge; additional copies may be obtained at cost in multiples of 50 .

The Pacific Journal of Mathematics is published quarterly, in March, June, September, and December. Effective with Volume 13 the price per volume (4 numbers) is $\$ 18.00$; single issues, $\$ 5.00$. Special price for current issues to individual faculty members of supporting institutions and to individual members of the American Mathematical Society: $\$ 8.00$ per volume; single issues $\$ 2.50$. Back numbers are available.

Subscriptions, orders for back numbers, and changes of address should be sent to Pacific Journal of Mathematics, 103 Highland Boulevard, Berkeley 8, California.

Printed at Kokusai Bunken Insatsusha (International Academic Printing Co., Ltd.), No. 6, 2-chome, Fujimi-cho, Chiyoda-ku, Tokyo, Japan.

PUBLISHED BY PACIFIC JOURNAL OF MATHEMATICS, A NON-PROFIT CORPORATION

The Supporting Institutions listed above contribute to the cost of publication of this Journal, but they are not owners or publishers and have no responsibility for its content or policies. 


\section{Pacific Journal of Mathematics}

\section{Vol. 12, No. $2 \quad$ February, 1962}

William George Bade and Robert S. Freeman, Closed extensions of the Laplace operator determined by a general class of boundary conditions . . . . . . . 395

William Browder and Edwin Spanier, H-spaces and duality ............. 411

Stewart S. Cairns, On permutations induced by linear value functions . . . . . . . 415

Frank Sydney Cater, On Hilbert space operators and operator roots of

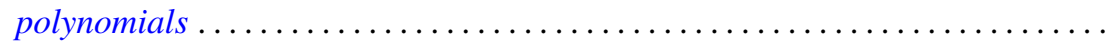

Stephen Urban Chase, Torsion-free modules over $K[x, y] \ldots \ldots \ldots \ldots \ldots \ldots \ldots 437$

Heron S. Collins, Remarks on affine semigroups . . . . . . . . . . . . . . . . 449

Peter Crawley, Direct decompositions with finite dimensional factors . . . . . . . 457

Richard Brian Darst, A continuity property for vector valued measurable

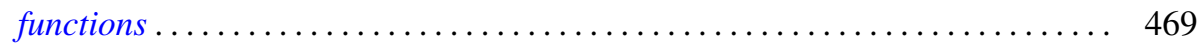

R. P. Dilworth, Abstract commutative ideal theory ................. 481

P. H. Doyle, III and John Gilbert Hocking, Continuously invertible spaces . . . . . . 499

Shaul Foguel, Markov processes with stationary measure . . . . . . . . . . . 505

Andrew Mattei Gleason, The abstract theorem of Cauchy-Weil ............ 511

Allan Brasted Gray, Jr., Normal subgroups of monomial groups . . . . . . . . . . 527

Melvin Henriksen and John Rolfe Isbell, Lattice-ordered rings and function

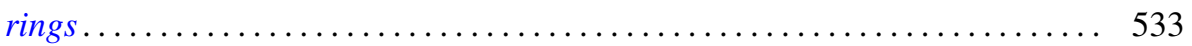

Amnon Jakimovski, Tauberian constants for the $[J, f(x)]$ transformations . ..... 567

Hubert Collings Kennedy, Group membership in semigroups . . . . . . . . . . . 577

Eleanor Killam, The spectrum and the radical in locally $m$-convex algebras ..... 581

Arthur H. Kruse, Completion of mathematical systems . . . . . . . . . . . . . 589

Magnus Lindberg, On two Tauberian remainder theorems ................ 607

Lionello A. Lombardi, A general solution of Tonelli's problem of the calculus of

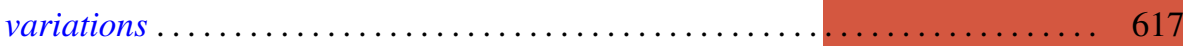

Marvin David Marcus and Morris Newman, The sum of the elements of the powers

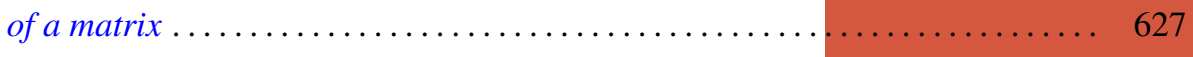

Michael Bahir Maschler, Derivatives of the harmonic measures in

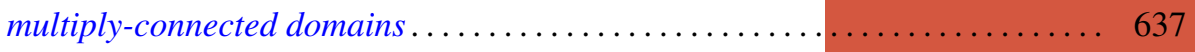

Deane Montgomery and Hans Samelson, On the action of $\mathrm{SO}(3)$ on $S^{n} \ldots \ldots \ldots 649$

J. Barros-Neto, Analytic composition kernels on Lie groups . . . . . . . . . . . . 661

Mario Petrich, Semicharacters of the Cartesian product of two semigroups ...... 679

John Sydney Pym, Idempotent measures on semigroups . . . . . . . . . . . . 685

K. Rogers and Ernst Gabor Straus, A special class of matrices . . . . . . . . . . . . 699

U. Shukla, On the projective cover of a module and related results . . . . . . . . . 709

Don Harrell Tucker, An existence theorem for a Goursat problem . . . . . . . . . . . 719

George Gustave Weill, Reproducing kernels and orthogonal kernels for analytic

differentials on Riemann surfaces ......................... 729

George Gustave Weill, Capacity differentials on open Riemann surfaces ........ 769

G. K. White, Iterations of generalized Euler functions . . . . . . . . . . . . . 777

Adil Mohamed Yaqub, On certain finite rings and ring-logics . . . . . . . . . 785 